\title{
ON THE EXTENSIONS OF THE DARBOUX THEORY OF INTEGRABILITY
}

\author{
JAUME GINÉ ${ }^{1}$, MAITE GRAU ${ }^{1}$ AND JAUME LLIBRE ${ }^{2}$
}

\begin{abstract}
Recently some extensions of the classical Darboux integrability theory to autonomous and non-autonomous polynomial vector fields have been done. The classical Darboux integrability theory and its recent extensions are based on the existence of algebraic invariant hypersurfaces. However the algebraicity of the invariant hypersurfaces is not necessary and the unique necessary condition is the algebraicity of the cofactors associated to them. In this note it is established a more general extension of the classical Darboux integrability theory.
\end{abstract}

\section{INTRODUCTION}

One of the classical problems in the theory of differential equations is to decide when a differential system is integrable or not. There is not a unique definition of integrability for a differential equation. Initially, the notion of "integrability" was introduced to describe the property of differential equations for which all local and global information can be obtained either explicitly from solutions or implicitly from invariants. The first class of invariants are the constants of motion, conserved quantities or first integrals. Of course there are also other invariants like integral invariants, integrating factors, Jacobi multipliers, Lax pairs or Lax operators, tensor invariants, or symmetries which give rise to different techniques for integrating differential equations, see for instance $[1,4,20,22,29,31]$ and references therein. The connections between these different techniques are, in general, unknown and these connections induce a very active research topic, see for example $[2,6,19]$.

In this note we focus on the integrability problem for differential systems and in this context the notion of integrability is based on the knowledge of first integrals which can be represented by the combination of known functions.

2010 Mathematics Subject Classification. 34C05, 34A34, 34C20.

Key words and phrases. nonlinear differential equations, integrability problem, first integral, invariant curves, exponential factors. 
In 1878 Darboux [9] presented a simple method to construct first integrals and integrating factors for planar polynomial vector fields using their invariant algebraic curves. In short he showed how first integrals of polynomial vector fields possessing sufficient invariant algebraic curves can be constructed. Specifically he proved that if a planar polynomial system of degree $m$ has at least $q=m(m+1) / 2$ invariant algebraic curves then it has a first integral or an integrating factor of the form $\prod_{i=1}^{q} f_{i}^{\lambda_{i}}(x, y)$ for suitable $\lambda_{i} \in \mathbb{C}$ not all zero and where $f_{i}(x, y)=0$ are invariant algebraic curves for $i=1, \ldots, q$. In 1979 Jouanolou [21] showed that if $q=m(m+1) / 2+2$ then the polynomial system has a rational first integral and consequently all its invariant curves are algebraic. In 1983 Prelle and Singer [30] proved that if a polynomial system has an elementary first integral, then this first integral can be computed by using the invariant algebraic curves of the system. In 1992 Singer [33] proved that if a polynomial system has a Liouvillian first integral, then it can be computed by using the invariant algebraic curves and the exponential factors of the system. Indeed Darboux [10] extended his method to polynomial vector fields in $\mathbb{C}^{n}$ by using invariant algebraic hypersurfaces. Recently some extensions of this classical method started by Darboux have been done by several authors taking into account exponential factors and the multiplicity of the invariant algebraic curves or hypersurfaces, see [8, 25, 26, 27] and references therein. In $[3,23]$ the Darboux theory of integrability is extended to non-autonomous polynomial vector fields in $\mathbb{C}^{n}$. More precisely in such works differential vector fields which are polynomials in the variables $x_{1}, x_{2}, \ldots, x_{n}$ with coefficients which are functions of the independent variable $t$ are considered.

All these extensions are based on the existence of invariant algebraic hypersurfaces. However the algebraicity of the invariant hypersurfaces is not necessary and the unique necessary condition is the algebraicity of the cofactors associated to them. In this note a more general extension of the classical Darboux integrability theory is established.

\section{EXtensions of the DARBoux THEORY OF INTEGRABILITY}

Let $U$ be an open subset of $\mathbb{C}$. We denote by $\mathcal{C}^{k}(U, \mathbb{C})$ the set of $\mathcal{C}^{k}$ functions from $U \rightarrow \mathbb{C}$ such that they do not vanish in $U$ except in a subset of Lebesgue measure zero and such that the closure of their domain of definition is $U$. In the subsequel, we assume that $k \geq 1$ is high enough so that all the considered computations can be done by at least continuous functions. We also consider the ring of polynomials in the variables $x_{1}, \ldots, x_{n}$ with coefficients in $\mathcal{C}^{k}(U, \mathbb{C})$ and we denote it 
by $\mathcal{C}^{k}(U, \mathbb{C})[x]=\mathcal{C}^{k}(U, \mathbb{C})\left[x_{1}, \ldots, x_{n}\right]$. We also denote by $\mathcal{C}^{k}(U, \mathbb{C})(x)$ the ring of rational functions in the variables $x_{1}, \ldots, x_{n}$ and coefficients in $\mathcal{C}^{k}(U, \mathbb{C})$.

In this note we consider complex polynomial differential systems in $\mathbb{C}^{n}$ of the form

$$
\dot{x}_{j}=P_{j}\left(t, x_{1}, \ldots, x_{n}\right)=\sum_{0 \leq i_{1}+\cdots+i_{n} \leq m} a_{j i_{1} \cdots i_{n}}(t) x_{1}^{i_{1}} \cdots x_{n}^{i_{n}},
$$

with $a_{j i_{1} \cdots i_{n}}(t) \in \mathcal{C}^{k}(U, \mathbb{C})$ and $P_{j} \in \mathcal{C}^{k}(U, \mathbb{C})[x]$ for $j=1, \ldots, n$. System (1) is a polynomial differential system in the variables $x_{1}, \ldots, x_{n}$ of degree $m=\max \left\{\operatorname{deg} P_{1}, \ldots, \operatorname{deg} P_{n}\right\}$ in $x_{1}, \ldots, x_{n}$. Note that in the particular case that the coefficients $a_{j i_{1} \cdots i_{n}}(t)$ are polynomials in the variable $t$ then adding the differential equation $\dot{t}=1$ we can apply the classical Darboux integrability theory to system (1). In the case that all the functions $P_{j}$ do not depend on $t$ we say that system (1) is autonomous. On the contrary we say that it is non-autonomous. Additionally, we associate with the differential system (1) the vector field

$$
\mathcal{X}=\frac{\partial}{\partial t}+\sum_{j=1}^{n} P_{j} \frac{\partial}{\partial x_{j}} \quad \text { or } \quad \mathcal{X}=\sum_{j=1}^{n} P_{j} \frac{\partial}{\partial x_{j}}
$$

in the non-autonomous case or the autonomous one, respectively. One can extend the Darboux integrability theory by considering invariant hypersurfaces $f_{i}=0$ of the form $f_{i} \in \mathcal{C}^{k}(U, \mathbb{C})[x]$ which satisfy $\mathcal{X}\left(f_{i}\right)=K_{i} f_{i}$ where $K_{i} \in \mathcal{C}^{k}(U, \mathbb{C})[x]$ is called the cofactor of the invariant hypersurface $f_{i}=0$. The works $[3,23]$ go in this direction. In what follows we call such invariant hypersurfaces polynomial invariant hypersurfaces. Another possible extension consists in considering exponential factors. Assume that $h, g \in \mathcal{C}^{k}(U, \mathbb{C})[x]$ are coprime in the variables $x_{1}, \ldots, x_{n}$. The function $F=\exp (g / h)$ is an exponential factor of system (1) if for some $L \in \mathcal{C}^{k}(U, \mathbb{C})[x]$ the following identity $\mathcal{X} F=L F$ is satisfied, where $L$ is called the cofactor of the exponential factor $F$. Finally, any function of the form

$$
f_{1}^{\lambda_{1}} \cdots f_{p}^{\lambda_{p}} \exp (g / h)
$$

is called a generalized Darboux function.

Let $W$ be an open subset of $U \times \mathbb{C}^{n}$, where $U$ is the open set of $\mathbb{C}$ considered at the beginning of the section. A function $H: W \rightarrow \mathbb{C}$ is a first integral of system (1) if $H$ is continuous, not locally constant and constant on each trajectory of the system (1) contained in $W$. We note that if $H$ is of class at least $\mathcal{C}^{1}$ in $W$, then $H$ is a first integral if it is not locally constant and $\mathcal{X}(H)=0$ on $W$. 
A function $M: W \rightarrow \mathbb{C}$ is a Jacobi multiplier of system (1) if $M$ is of class $\mathcal{C}^{1}$, not locally zero and satisfies $\mathcal{X}(M)=-(\operatorname{div} \mathcal{X}) M$ where $\operatorname{div} \mathcal{X}=\sum_{i=1}^{n} \partial P_{i} / \partial x_{i}$ is the divergence of system (1).

For systems (1) a Darboux first integral is a first integral given by a generalized Darboux function (2), and in a similar way we define a Darboux Jacobi multiplier.

The extensions of the Darboux integrability theory for differential systems are established in Theorem 2 in [23] and Theorem 8 in [3]. Both extensions are based in the computation of the rank of the Wronskian matrix of the cofactors corresponding to the invariant hypersurfaces and to the exponential factors. More precisely consider $K_{1}, \ldots, K_{r}$ cofactors of the invariant hypersurfaces or of the exponential factors of $\mathcal{X}$, and denote by $\mathcal{W}$ the Wronskian matrix of the cofactors defined as

$$
\mathcal{W}=\mathcal{W}\left(K_{1}, \ldots, K_{r}\right)=\left(\begin{array}{ccc}
K_{1} & \ldots & K_{r} \\
\mathcal{X}\left(K_{1}\right) & \ldots & \mathcal{X}\left(K_{r}\right) \\
& \ldots & \\
\mathcal{X}^{(r-1)}\left(K_{1}\right) & \ldots & \mathcal{X}^{(r-1)}\left(K_{r}\right)
\end{array}\right)
$$

where $\mathcal{X}^{(l+1)}\left(K_{i}\right)=\mathcal{X}\left(\mathcal{X}^{(l)}\left(K_{i}\right)\right)$. In the following section we improve these extensions taking into account that it is not necessary that the functions that define the invariant hypersurfaces $f_{i}$ or the exponentials factors $h$ and $g$ be polynomials in the variables $x_{1}, x_{2}, \ldots, x_{n}$, that is, it is not necessary the algebraicity of these functions with respect to the variables $x_{1}, x_{2}, \ldots, x_{n}$ and the unique necessary condition is the algebraicity of the cofactors associated to them.

We shall need the following result which is Lemma 14 proved in [23].

Let $S=\left\{K_{1}, \cdots, K_{p}\right\}$ be a set of polynomials of $\mathcal{C}^{k}(U, \mathbb{C})[x]$ and let $S_{r}$ be a subset of $S$ of $r$ elements. We denote by $\mathcal{W}\left(S_{r}\right)$ the Wronskian of these $r$ elements. We write $\mathcal{W}_{r}=0$ if for all subsets $S_{r}$ of $S$ we have that $\mathcal{W}\left(S_{r}\right) \equiv 0$ for all $t \in U$. If for some subset $S_{r}$ we have that $\mathcal{W}\left(S_{r}\right) \neq 0$ for some $t \in U$, then we write $\mathcal{W}_{r} \neq 0$. We say that the set of the polynomials of $S$ satisfies condition $\mathcal{W}^{*}$ if there exists $s \in\{2,3, \cdots, p\}$ such that $\mathcal{W}_{j} \neq 0$ for $j=1, \cdots, s-1$ and $\mathcal{W}_{s}=0$.

Lemma 1. Assume that $K_{1}, \cdots, K_{r} \in \mathcal{C}^{k}(U, \mathbb{C})[x] \backslash\{0\}$.

(a) If there is $\left(t_{0}, x_{0}\right) \in U \times \mathbb{C}^{n}$ such that $\mathcal{W}_{r} \neq 0$ at $\left(t_{0}, x_{0}\right)$, then $K_{1}, \cdots, K_{r}$ are linearly independent over $\mathbb{C}$.

(b) If the set $\left\{K_{1}, \cdots, K_{r}\right\}$ satisfies the condition $\mathcal{W}^{*}$ for some $s \in$ $\{2,3, \cdots, r\}$, then there exists a subset of $s$ elements linearly dependent over $\mathbb{C}$. 


\section{A MORE GENERAL EXTENSiON}

Let $U$ be a connected open subset of $\mathbb{C} \times \mathbb{C}^{n}$. We consider $\mathcal{C}^{k}(U, \mathbb{C} \times$ $\left.\mathbb{C}^{n}\right)$ the ring of functions of class $\mathcal{C}^{k}$ with respect all its variables $t$, $x_{1}, x_{2}, \ldots, x_{n}$. Now we deal with a differential system of the form (1) and we consider invariant hypersurfaces $f_{i} \in \mathcal{C}^{k}\left(U, \mathbb{C} \times \mathbb{C}^{n}\right)$ and exponential factors $F=\exp (g / h)$ where $g, h \in \mathcal{C}^{k}\left(U, \mathbb{C} \times \mathbb{C}^{n}\right)$. However for each invariant hypersurface and exponential factor we assume that their cofactors $K_{i} \in \mathcal{C}^{k}(U, \mathbb{C})[x]$ (the ring of polynomials in the variables $x_{1}, x_{2}, \ldots, x_{n}$ with coefficients in $\mathcal{C}^{k}(U, \mathbb{C})$ ). The following statement generalizes the results given in $[3,23]$. Indeed, the following theorem provides an extension of the Darboux theory of integrability for $n$-dimensional autonomous or non-autonomous differential systems.

Theorem 2. We assume that the differential system (1) of degree $m$ admits

(i) $p$ invariant hypersurfaces $f_{1}=0, \ldots, f_{p}=0$ with cofactors $K_{1}, \ldots, K_{p}$, where $f_{1}, \ldots, f_{p} \in \mathcal{C}^{k}\left(U, \mathbb{C} \times \mathbb{C}^{n}\right)$ and $K_{1}, \ldots, K_{p} \in$ $\mathcal{C}^{k}(U, \mathbb{C})[x]$

(ii) $q$ exponential factors $F_{p+1}, \ldots, F_{p+q}$ with cofactors $K_{p+1}, \ldots$, $K_{p+q}$ where each $F_{i}=\exp \left(g_{i} / h_{i}\right)$ and $g_{i}, h_{i} \in \mathcal{C}^{k}\left(U, \mathbb{C} \times \mathbb{C}^{n}\right)$ and $K_{p+1}, \ldots, K_{p+q} \in \mathcal{C}^{k}(U, \mathbb{C})[x]$.

We further assume that $d \log f_{1}, \ldots, d \log f_{p}, d \log F_{p+1}, \ldots, d \log F_{p+q}$ are $\mathbb{C}$-linearly independent as $\mathcal{C}^{k-1} 1$-forms in $U \subseteq \mathbb{C} \times \mathbb{C}^{n}$. Then the following statements hold.

(a) $p+q>\operatorname{rank}\left(\mathcal{W}\left(K_{1}, \ldots, K_{p+q}\right)\right)$ if and only if there is a first integral. This first integral is a generalized Darboux first integral of the form (2).

(b) $p+q>\operatorname{rank}\left(\mathcal{W}\left(K_{1}, \ldots, K_{p+q}\right.\right.$, div $\left.\left.\mathcal{X}\right)\right)$ if and only if there is a Darboux Jacobi multiplier of $\mathcal{X}$ of the form (2).

Proof. By hypothesis we have $p$ invariant hypersurfaces $f_{i}=0$ with cofactors $K_{i}$, and $q$ exponential factors $F_{p+j}$ with cofactors $K_{p+j}$. That is, the $f_{i}$ 's satisfy $\mathcal{X} f_{i}=K_{i} f_{i}$, and the $F_{p+j}$ 's satisfy $\mathcal{X} F_{p+j}=K_{p+j} F_{p+j}$.

We have the following equalities

$$
\begin{array}{r}
\mathcal{X}\left(f_{1}^{\lambda_{1}} \ldots f_{p}^{\lambda_{p}} F_{p+1}^{\mu_{1}} \ldots F_{p+q}^{\mu_{q}}\right)= \\
\left(f_{1}^{\lambda_{1}} \ldots f_{p}^{\lambda_{p}} F_{p+1}^{\mu_{1}} \ldots F_{p+q}^{\mu_{q}}\right)\left(\sum_{i=1}^{p} \lambda_{i} \frac{\mathcal{X} f_{i}}{f_{i}}+\sum_{j=1}^{q} \mu_{j} \frac{\mathcal{X} F_{p+j}}{F_{p+j}}\right)= \\
\left(f_{1}^{\lambda_{1}} \ldots f_{p}^{\lambda_{p}} F_{p+1}^{\mu_{1}} \ldots F_{p+q}^{\mu_{q}}\right)\left(\sum_{i=1}^{p} \lambda_{i} K_{i}+\sum_{j=1}^{q} \mu_{j} K_{p+j}\right) .
\end{array}
$$


We prove statement (a). Since $p+q>\operatorname{rank}\left(\mathcal{W}\left(K_{1}, \ldots, K_{p+q}\right)\right)$, then by Lemma 1 there is a non-trivial linear combination over $\mathbb{C}$ of the cofactors $K_{1}, \ldots, K_{p+q}$, that is $\lambda_{1} K_{1}+\cdots+\lambda_{p} K_{p}+\mu_{1} K_{p+1}+\cdots+$ $\mu_{p+q} K_{p+q}=0$. Therefore, from (3) it follows that

$$
\mathcal{X}\left(f_{1}^{\lambda_{1}} \ldots f_{p}^{\lambda_{p}} F_{p+1}^{\mu_{1}} \ldots F_{p+q}^{\mu_{q}}\right)=0 .
$$

So $f_{1}^{\lambda_{1}} \ldots f_{p}^{\lambda_{p}} F_{p+1}^{\mu_{1}} \ldots F_{p+q}^{\mu_{q}}$ is a first integral of system (1). Moreover, this function is not a constant in $U \subseteq \mathbb{C} \times \mathbb{C}^{n}$ because of the linear $\mathbb{C}$-independence of $d \log f_{1}, \ldots, d \log f_{p}, d \log F_{p+1}, \ldots, d \log F_{p+q}$.

Conversely, if the function $f_{1}^{\lambda_{1}} \ldots f_{p}^{\lambda_{p}} F_{p+1}^{\mu_{1}} \ldots F_{p+q}^{\mu_{q}}$ is a first integral of system (1), then $\mathcal{X}\left(f_{1}^{\lambda_{1}} \ldots f_{p}^{\lambda_{p}} F_{p+1}^{\mu_{1}} \ldots F_{p+q}^{\mu_{q}}\right)=0$. Hence, from (3) it follows that $\lambda_{1} K_{1}+\cdots+\lambda_{p} K_{p}+\mu_{1} K_{p+1}+\cdots+\mu_{p+q} K_{p+q}=0$. Therefore, by Lemma 1 we have that $p+q>\operatorname{rank}\left(\mathcal{W}\left(K_{1}, \ldots, K_{p+q}\right)\right)$. So statement (a) is proved.

We have the following equalities

$$
\begin{aligned}
\mathcal{X}\left(f_{1}^{\lambda_{1}} \ldots f_{p}^{\lambda_{p}} F_{p+1}^{\mu_{1}} \ldots F_{p+q}^{\mu_{q}}\right) & = \\
\left(f_{1}^{\lambda_{1}} \ldots f_{p}^{\lambda_{p}} F_{p+1}^{\mu_{1}} \ldots F_{p+q}^{\mu_{q}}\right)\left(\sum_{i=1}^{p} \lambda_{i} \frac{\mathcal{X} f_{i}}{f_{i}}+\sum_{j=1}^{q} \mu_{j} \frac{\mathcal{X} F_{p+j}}{F_{p+j}}\right) & = \\
\left(f_{1}^{\lambda_{1}} \ldots f_{p}^{\lambda_{p}} F_{p+1}^{\mu_{1}} \ldots F_{p+q}^{\mu_{q}}\right)\left(\sum_{i=1}^{p} \lambda_{i} K_{i}+\sum_{j=1}^{q} \mu_{j} K_{p+j}\right) & = \\
-\left(f_{1}^{\lambda_{1}} \ldots f_{p}^{\lambda_{p}} F_{p+1}^{\mu_{1}} \ldots F_{p+q}^{\mu_{q}}\right) \operatorname{div}(\mathcal{X}) . &
\end{aligned}
$$

Now using the previous equalities the proof of statement (b) follows using the same kind of arguments than in the proof of statement (a).

Remark 3. It is important to note that in Theorem 2 there is no condition about the degree of the cofactors $K_{1}, \ldots, K_{p+q}$.

From the results of [33] it follows that the Darboux integrability theory for autonomous polynomial differential systems finds all the Liouvillian first integrals. Nevertheless there are polynomial differential systems with non-Liouvillian first integral and some of them can be detected with the more general extension of the Darboux integrability theory given in the present work. The following simple example shows this fact. The example corresponds to one autonomous system but it is also generalizable to non-autonomous systems.

Example 4. We consider the polynomial differential system

$$
\dot{x}=-y+a x^{n}+x^{2 n}, \quad \dot{y}=b x^{n-1},
$$


where $n \geq 1$ is a positive integer. From Odani's result [28], it easily follows that system (5) does not have any finite invariant algebraic curve. The existence of finite invariant algebraic curves for an arbitrary planar differential system is made in $[15,17,18]$. From Singer's results system (5) is Liouvillian integrable if it admits an integrating factor of the form (2). Hence, the only possible generalized Darboux integrating factor that admits system (5), if it exists, is an exponential factor of the form $\exp (h)$ with $h \in \mathbb{C}[x, y]$. However, from the definition of integrating factor we have $\mathcal{X} \exp (h)=-\operatorname{div} \mathcal{X} \exp (h)$, that is

$$
\left(-y+a x^{n}+x^{2 n}\right) \frac{\partial h}{\partial x}+b x^{n-1} \frac{\partial h}{\partial y}=-a n x^{n-1}-2 n x^{2 n-1},
$$

where we have simplified the common factor $\exp (h)$. Let $h(x, y)=$ $\sum_{i=0}^{N} h_{i}(y) x^{i}$, where $h_{i}(y) \in \mathbb{C}[y]$ with $h_{N}(y) \not \equiv 0$. Equating the highest degree terms in both members of (6) we obtain $N h_{N}(y) x^{N+2 n-1}=0$. This implies $N=0$ which is a contradiction. Therefore (5) does not have any Liouvillian first integral. This example is a generalization of the example presented in [12]. System (5) has a non-Liouvillian first integral $H(x, y)=f_{1} f_{2}^{-1}$, with $f_{1}(x, y)=n^{1 / 3}\left(a+2 x^{n}\right) \operatorname{Ai}(\xi)+$ $2 b^{1 / 3} \mathrm{Ai}^{\prime}(\xi)$ and $f_{2}(x, y)=n^{1 / 3}\left(a+2 x^{n}\right) \operatorname{Bi}(\xi)+2 b^{1 / 3} \operatorname{Bi}^{\prime}(\xi)$, and $\xi=$ $\left(n^{2} / b^{2}\right)^{1 / 3}\left(a^{2}+4 y\right) / 4$, where $f_{1}=0$ and $f_{2}=0$ are invariant curves with polynomial cofactors $K_{1}=K_{2}=n x^{n-1}\left(a+2 x^{n}\right) / 2$. Here $\operatorname{Ai}(x)$ and $\operatorname{Bi}(x)$ are the pair of linearly independent solutions of the Airy equation $\omega^{\prime \prime}=x \omega$. Moreover an integrating factor is given by $M=f_{1}^{-2}(x, y)$ with cofactor $-\operatorname{div} \mathcal{X}=-n a x^{n-1}-2 n x^{2 n-1}$.

To have an algorithm for detecting non-polynomial invariant curves or hypersurfaces is the first goal in order to apply the extension of the Darboux theory of integrability given in this work. In fact in [13] it is given the first method to detect non-algebraic invariant curves for polynomial planar vector fields. This approach is based on the existence of a polynomial cofactor for such curves. As an application of this algorithmic method, some Lotka-Volterra systems with non-algebraic invariant curves are given.

The second important question is whether any non-polynomial invariant hypersurfaces has always a polynomial cofactor. The answer to this question is negative. There are examples of non-polynomial invariant curves or hypersurfaces with a cofactor which is not polynomial as the following simple example shows.

Example 5. Consider the polynomial differential system

$$
\dot{x}=2 y, \quad \dot{y}=x-y^{4} .
$$


System (7) has the non-algebraic invariant curve $f=0$ where $f=$ $y+\sqrt{\operatorname{Ai}(x) / \mathrm{Ai}^{\prime}(x)}$, with the non-polynomial cofactor

$K(x, y)=-y^{3}+y^{2} \sqrt{\operatorname{Ai}(x) / \operatorname{Ai}^{\prime}(x)}-y \operatorname{Ai}(x) / \operatorname{Ai}^{\prime}(x)+x \sqrt{\operatorname{Ai}^{\prime}(x) / \operatorname{Ai}(x)}$.

Moreover a formal invariant curve $f(x, y)=0$ of a planar autonomous differential system given by a formal power expansion $f \in \mathbb{C}[[x, y]]$ must satisfy an equation $\mathcal{X} f=L f$ where $L \in \mathbb{C}[[x, y]]$ is also a formal power expansion, see $[7,32]$. The form of the non-polynomial cofactor of example 5 suggests to define the so-called quasipolynomial cofactor given in [11], where the following result was established.

Proposition 6. Any invariant curve $f=y-g(x)=0$ of a planar polynomial autonomous differential system has a unique quasipolynomial cofactor of the form

$$
K(x, y)=K_{m-1}(x) y^{m-1}+\cdots+K_{1}(x) y+K_{0}(x),
$$

where $m$ is the degree of the autonomous system (1) with $n=2$.

As a consequence of Proposition 6 we can estimate the form of the cofactors of some non-polynomial invariant curves or hypersurfaces. In order to do that we first introduce some notation.

As usual we define $\mathbb{C}[[x]]$ the set of the formal power series in the variable $x$ with coefficients in $\mathbb{C}$ and $\mathbb{C}[y]$ the set of the polynomials in the variable $y$ with coefficients in $\mathbb{C}$. A polynomial of the form

$$
\sum_{i=0}^{\ell} a_{i}(x) y^{i} \in \mathbb{C}[[x]][y]
$$

is called a formal Weierstrass polynomial in $y$ of degree $\ell$. A formal Weierstrass polynomial whose coefficients are convergent is called a Weierstrass polynomial. We observe that a quasipolynomial cofactor is in fact a formal Weierstrass polynomial. Now we can estimate the form of the cofactor of any formal Weierstrass polynomial.

Proposition 7. Any invariant curve $f=0$ of a planar polynomial autonomous differential system which is a formal Weierstrass polynomial of degree $\ell$, that is $f$ is of the form $f(x, y)=\sum_{i=0}^{\ell} a_{i}(x) y^{i} \in \mathbb{C}[[x]][y]$, has a formal Weierstrass polynomial cofactor of degree at most $m-1$ where $m$ is the degree of the autonomous system (1) with $n=2$.

In order to prove this proposition we need some notation and several results, following [5]. We recall that $\mathbb{C}((x))$ denotes the field of fractions of $\mathbb{C}[[x]]$. Given $d \in \mathbb{N}$, we consider entire fractional series $s=\sum_{i \geq r} a_{i} x^{i / d}$ where $r \in \mathbb{Z}, a_{i} \in \mathbb{C}$ and $\min \left\{i: a_{i} \neq 0\right\} \geq 0$. An 
element $s \in \mathbb{C}\left(\left(x^{1 / d}\right)\right)$ is of the form $s=\sum_{i>r} a_{i} x^{i / d}$ where $r \in \mathbb{Z}$, $a_{i} \in \mathbb{C}$ and the $\min \left\{i: a_{i} \neq 0\right\}$ can be lower than zero. The elements of the ring $\mathbb{C}\left[\left[x^{1 / d}\right]\right]$ are the entire fractional series such that $\min \left\{i: a_{i} \neq 0\right\} \geq 0$. An element $s \in \mathbb{C}\left[\left[x^{1 / d}\right]\right]$ is called a Puiseux series. The fractional series $s$ is said to be convergent if $\sum_{i \geq r} a_{i} t^{i}$ has non-zero convergence radius, where $t=x^{1 / n}$. We need the following statement which is Corollary 1.5.6 of [5].

Lemma 8. If $f \in \mathbb{C}[[x, y]]$ then it has a unique decomposition of the form

$$
f=u x^{r} \prod_{j=1}^{\ell}\left(y-g_{j}(x)\right),
$$

where $g_{j}(x)$ are Puiseux series and $r \in \mathbb{Z}, r \geq 0$ and $u \in \mathbb{C}[[x, y]]$ is invertible inside the ring $\mathbb{C}[[x, y]]$.

Since we are considering a formal Weierstrass polynomial $f(x, y)$ instead of a formal series, we deduce that $\ell$ is the highest degree in $y$ of $f(x, y)$ and, therefore, $u$ is an invertible power series of $\mathbb{C}[[x]]$. We define $h(x)=u x^{r}$ and we have that given $f(x, y) \in \mathbb{C}[[x]][y]$ of degree $\ell$ in $y$, there is a unique decomposition of the form:

$$
f=h(x) \prod_{i=1}^{\ell}\left(y-g_{i}(x)\right),
$$

where $h(x) \in \mathbb{C}[[x]]$ and $g_{i}(x)$ are Puiseux series.

Proof of Proposition 7. We consider an autonomous differential system (1) with $n=2$ and we are assuming that $f$ is an invariant curve. By the decomposition (9) of $f$ we have that $h(x)=0$, if $h$ is not a constant, is an invariant curve and that $y-g_{i}(x)=0$ are invariant curves of the system for $i=1,2, \ldots, \ell$. Our planar differential system is of the form

$$
\dot{x}=P(x, y), \quad \dot{y}=Q(x, y),
$$

where $P(x, y)$ and $Q(x, y)$ are polynomials of degree at most $m$. If $h$ is not a constant, then the polynomial $P(x, y)$ needs to have degree at most $m-1$ in the variable $y$. The cofactor of the invariant curve $h(x)=0$ is $P(x, y) h^{\prime}(x) / h(x)$ which is a formal Weierstrass polynomial cofactor polynomial in $y$ of degree at most $m-1$. By Proposition 6 , each of the invariant curves $y-g_{i}(x)=0$, for $i=1,2, \ldots, \ell$, has a unique formal Weierstrass polynomial cofactor which is a polynomial in $y$ of degree at most $m-1$. Therefore, since $f$ is the product of 
all these invariant curves, then its cofactor is the sum of all their formal Weierstrass polynomial cofactors which forms a formal Weierstrass polynomial cofactor of degree at most $m-1$ in $y$.

The generalizations of the more general extension of the Darboux integrability theory given in this paper are based in the concept of Weierstrass polynomial and its Weierstrass polynomial cofactor. Indeed in [14] is given a first generalization based in the following definition. A planar autonomous differential system is Weierstrass integrable if admits an integrating factor of the form

$$
M=\exp \{D / E\} \prod C_{i}^{l_{i}},
$$

where $D, E$, and the $C_{i}$ are Weierstrass polynomials and $l_{i} \in \mathbb{C}$.

Liouvillian integrable systems which have a Darboux integrating factor of form (2), are included in the set of systems which are Weierstrass integrable. However, there are systems which are Weierstrass integrable which are not Liouvillian integrable, see for instance Example 4 of the present paper. In [16, 24] Liénard systems and Abel equations that are Weierstrass integrable are studied.

\section{ACKnowledgements}

The first and second authors are partially supported by a MICINN/ FEDER grant number MTM2011-22877 and by a AGAUR (Generalitat de Catalunya) grant number 2009SGR 381. The third author is partially supported by a MICINN/ FEDER grant number MTM200803437, by a AGAUR grant number 2009SGR 410, by ICREA Academia and by FP7-PEOPLE-2012-IRSES numbers 316338 and 318999.

\section{REFERENCES}

[1] M.J. Ablowitz, A. Ramani And H. Segur, A connection between nonlinear evolution equations and ordinary differential equations of P-type $I$ and II, J. Math. Phys. (1980) 21, 715-721 and 1006-1015.

[2] A. BAIDER, Completion of vector fields and the Painlevé property, J. Differential Equations 97 (1992), no. 1, 27-53.

[3] D. Blázquez-Sanz and C. Pantazi, A note on the Darboux theory of integrability of non-autonomous polynomial differential systems, Nonlinearity $\mathbf{2 5}$ (2012), 2615-2624.

[4] L.R. Berrone and H. Giacomini, Inverse Jacobi multipliers, Rend. Circ. Mat. Palermo II 52 (2003) 77-130.

[5] E. Casas-Alvero, Singularities of Plane Curves. London Mathematical Society Lecture Note Series 276 Cambridge University Press, Cambridge, 2000.

[6] D.D. Hua, L. Cairó, M.R. Feix, K.S. Govinder and P.G.L. Leach, Connection between the existence of first integrals and the Painlevé property in 
two-dimensional Lotka-Volterra and quadratic systems, Proc. Roy. Soc. London Ser. A 452 (1996), 859-880.

[7] J. Chavarriga, H. Giacomini, J. Giné and J. Llibre, Local analytic integrability for nilpotent centers, Ergodic Theory Dynam. Systems 23 (2003), 417-428.

[8] C. Christopher, J. Llibre and J. V. Pereira, Multiplicity of invariant algebraic curves in polynomial vector fields, Pacific J. Math. 229 (2007), 63117.

[9] G. Darboux, Mémoire sur les équations différentielles algébrique du premier ordre et du premier degré (Mélanges), Bull. Sci. Math. 2ème série, 2 (1878), 60-96; 123-144; 151-200.

[10] G. Darboux, De l'emploi des solutions particulières algébriques dans l'intégration des systèmes d'équations différentielles algébriques, C. R. Math. Acad. Sci. Paris 86 (1878), 1012-1014.

[11] I.A. García, H. Giacomini and J. Giné, Generalized nonlinear superposition principles for polynomial planar vector fields, J. Lie Theory 15 (2005), 89-104.

[12] I.A. García AND J. Giné, Generalized cofactos and nonlinear superposition principles, Appl. Math. Lett. 16 (2003), 1137-1141.

[13] I.A. García AND J. Giné, Non-algebraic invariant curves for polynomial planar vector fields, Discrete Contin. Dyn. Syst. 10 (2004), 755-768.

[14] J. Giné And M. Grau, Weierstrass integrability of differential equations, Appl. Math. Lett. 23 (2010), 523-526.

[15] J. Giné, M. Grau And J. Llibre, A note on Liouvillian first integrals and invariant algebraic curves, Appl. Math. Lett. 26 (2013), 285-289.

[16] J. Giné AND J. LliBRE, Weierstrass integrability in Liénard differential systems, J. Math. Anal. Appl. 377 (2011), 362-369.

[17] J. Giné And J. Llibre, A note on Liouvillian integrability, J. Math. Anal. Appl. 387 (2012), 1044-1049.

[18] J. Giné AND J. LliBRe, On Liouvillian integrability of the first-order polynomial ordinary differential equations J. Math. Anal. Appl. 395 (2012), 802-805.

[19] A. GoRIELY, Integrability, partial integrability, and nonintegrability for systems of ordinary differential equations, J. Math. Phys. 37 (1996), 1871-1893.

[20] A. Goriely, Integrability and nonintegrability of dynamical systems. Advanced Series in Nonlinear Dynamics, 19. World Scientific Publishing Co., Inc., River Edge, NJ, 2001

[21] J.P. Jounnolou, Equations de Pfaff algébriques, Lecture Notes in Mathematics 708, Springer-Verlag, 1979.

[22] P.D. LAX, Integrals of nonlinear equations of evolution and solitary waves, Commun. Pure Appl. Math. 21 (1968), 467-490.

[23] J. Llibre and Ch. Pantazi, Darboux theory of integrability for a class of nonautonomous vector fields, J. Math. Phys. 50 (2009), 102705.

[24] J. Llibre AND C. VAlls, Generalized Weierstrass integrability of the Abel differential equations, to appear in Mediterranean Journal of Mathematics, 2012.

[25] J. Llibre And X. Zhang, Darboux theory of integrability in $\mathbb{C}^{n}$ taking into account the multiplicity, J. Differential Equations 246 (2009), 541-551. 
[26] J. Llibre AND X. ZHANG, Darboux theory of integrability for polynomial vector fields in $\mathbb{R}^{n}$ taking into account the multiplicity at infinity, Bull. Sci. Math. 133 (2009), 765-778.

[27] J. LliBRe AND X. ZhANG, Rational first integrals in the Darboux theory of integrability in $\mathbb{C}^{n}$, Bull. Sci. Math. 134 (2010), 189-195.

[28] K. Odani, The limit cycle of the van der Pol equation is not algebraic, J. Differential Equations 115 (1995), 146-152.

[29] P.J. OLver, Applications of Lie groups to differential equations, Springer, New York, 1986.

[30] M.J. Prelle And M.F. Singer, Elementary first integrals of differential equations, Trans. Amer. Math. Soc. 279 (1983), 215-229.

[31] W. Sarlet and F. Cantrijn, Generalizations of Noether's theorem in classical mechanics, SIAM Rev. 23 (1981), 467-494.

[32] A. Seidenberg, Reduction of singularities of the differential equation $A d y=$ $B d x$, Amer. J. Math. 90 (1968), 248-269.

[33] M.F. Singer, Liouvillian first integrals of differential equations, Trans. Amer. Math. Soc. 333 (1992), 673-688.

1 Departament de Matemàtica, Universitat de Lleida, Avda. Jaume II, 69; 25001 Lleida, Catalonia, Spain

E-mail address: gine@matematica.udl.cat, mtgrau@matematica.udl.cat

2 Departament de Matemàtiques, Universitat Autònoma de Barcelona, 08193 Bellaterra, Barcelona, Catalonia, Spain

E-mail address: jllibre@mat.uab.cat 